\title{
Square Divisor Cordial Graphs
}

\author{
S. Murugesan \\ Department of Mathematics \\ C. B. M. College, Kovaipudur, \\ Coimbatore - 641042 , \\ Tamil Nadu, INDIA.
}

\author{
D. Jayaraman \\ Department of Mathematics \\ C. B. M. College, Kovaipudur, \\ Coimbatore - 641 042, Tamil Nadu, INDIA.
}

\author{
J. Shiama \\ Department of Mathematics, \\ C. M. S. College of Science and Commerce, \\ Coimbatore- 641 049, Tamil Nadu, INDIA.
}

\begin{abstract}
A square divisor cordial labeling of a graph $\mathrm{G}$ with vertex set $\mathrm{V}$ is a bijection $f$ from $\mathrm{V}$ to $\{1,2, \ldots,|V|\}$ such that if each edge $u v$ is assigned the label 1 if $[f(u)]^{2} \mid f(v)$ or $[f(v)]^{2} \mid f(u)$ and 0 otherwise, then the number of edges labeled with 0 and the number of edges labeled with 1 differ by atmost 1 . If a graph has a square divisor cordial labeling, then it is called square divisor cordial graph. In this paper, we investigate the square divisor cordial labeling behaviour of paths, cycles, wheel graphs, star graphs, some complete bipartite graphs and complete graphs.
\end{abstract}

\section{Keywords:}

Cordial labeling, square divisor cordial labeling, square divisor cordial graphs.

\section{INTRODUCTION}

By a graph $G$, we mean a finite, undirected graph without loops and multiple edges, for terms not defined here, we refer to Harary [5].

First we give the concept in number theory [3].

Definition 1. Let $a$ and $b$ be two integers. We say that a divides $b$ if there is a positive integer $k$ such that $b=k a$. It is denoted by $a \mid b$.

If a does not divide $b$, then we denote $a \nmid b$.

Graph labeling [4] is a strong communication between number theory [3] and structure of graphs [5]. By combining the divisibility concept in number theory and cordial labeling concept in graph labeling, we introduce a new concept called square divisor cordial labeling. In this paper, we prove the standard graphs such as paths $P_{n}(n \leq 12)$, cycles $C_{n}(3 \leq \mathrm{n} \leq 11)$, wheels, some star graphs, some complete bipartite graphs and some complete graphs are square divisor cordial.

A vertex labeling [4] of a graph $G$ is an assignment $f$ of labels to the vertices of $G$ that induces for each edge $u v$ a label depending on the vertex label $f(u)$ and $f(v)$. The two best known labeling methods are graceful and harmonius labelings. Cordial labeling is a variation of both graceful and harmonius labelings [1].

Definition 2. Let $G=(V, E)$ be a graph. A mapping $f$ : $V(G) \rightarrow\{0,1\}$ is called binary vertex labeling of $G$ and $f(v)$ is called the label of the vertex $v$ of $G$ under $f$.

For an edge $e=u v$, the induced edge labeling $f^{*}: E(G) \rightarrow\{0,1\}$ is given by $f^{*}(e)=|f(u)-f(v)|$. Let $v_{f}(0), v_{f}(1)$ be the number of vertices of $G$ having labels 0 and 1 respectively under $f$ and $e_{f}(0), e_{f}(1)$ be the number of edges having labels 0 and 1 respectively under $f^{*}$.

The concept of cordial labeling was introduced by Cahit [1].

Definition 3. A binary vertex labeling of a graph $G$ is called a cordial labeling if $\left|v_{f}(0)-v_{f}(1)\right| \leq 1$ and $\left|e_{f}(0)-e_{f}(1)\right| \leq$ 1. A graph $G$ is cordial if it admits cordial labeling.

Cahit proved some results in [2].

\section{MAIN RESULTS}

Sundaram, Ponraj and Somasundaram [6] have introduced the notion of prime cordial labeling.

Definition 4. [6] A prime cordial labeling of a graph $G$ with vertex set $V$ is a bijection $f$ from $V$ to $\{1,2, \ldots,|V|\}$ such that if each edge uv is assigned the label 1 if $g c d(f(u), f(v))=$ 1 and 0 if $g c d(f(u), f(v))>1$, then the number of edges labeled with 1 and the number of edges labeled with 0 differ by atmost 1 .

In [6], they have proved some graphs are prime cordial.

Varatharajan, Navaneethakrishnan and Nagarajan [7] have introduced the notion of divisor cordial labeling.

Definition 5. [7 Let $G=(V, E)$ be a simple graph and $f: V \rightarrow 1,2, \ldots,|V|$ be a bijection. For an edge uv, assign the label 1 if either $f(u) \mid f(v)$ or $f(v) \mid f(u)$ and the label 0 if $f(u) \nmid f(v)$. $f$ is called a divisor cordial labeling if $\left|e_{f}(0)-e_{f}(1)\right| \leq 1$.

A graph with a divisor cordial labeling is called a divisor cordial graph.

In [7], they have proved the standard graphs such as path, cycle, wheel, star and some complete bipartite graphs are divisor cordial. They also proved that complete graph is not divisor cordial. In [8], they have proved some special classes of graphs such as dragon, corona, full binary trees, $G * K_{2, n}$ and $G * K_{3, n}$ are divisor cordial. Motivated by the concept of divisor cordial labeling, we introduce a new special type of cordial labeling called square divisor cordial labeling as follows.

Definition 6. Let $G=(V, E)$ be a simple graph and $f$ : $V \rightarrow\{1,2, \ldots,|V|\}$ be a bijection. For each edge uv, assign the label 1 if either $[f(u)]^{2} \mid f(v)$ or $[f(v)]^{2} \mid f(u)$ and the label 0 otherwise. $f$ is called a square divisor cordial labeling if $\left|e_{f}(0)-e_{f}(1)\right| \leq 1$.

A graph with a square divisor cordial labeling is called a square divisor cordial graph.

Example 1.

Consider the following graph $G$. 


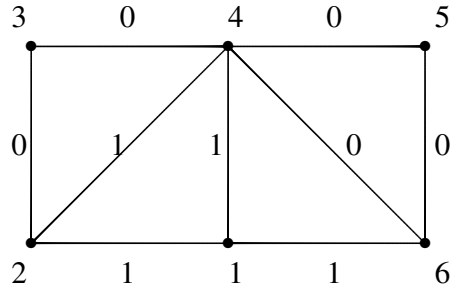

We see that $e_{f}(0)=5$ and $e_{f}(1)=4$. Thus $\left|e_{f}(0)-e_{f}(1)\right|=$ 1 and hence $\mathrm{G}$ is square divisor cordial.

Theorem 1. Given a positive integer $n$, there is a square divisor cordial graph $G$ which has $n$ vertices.

PROOF. Suppose $n$ is even. By constructing a path containing $\frac{n}{2}+2$ vertices $v_{1}, v_{2}, \ldots, v \frac{n}{2}+2$ which are labeled as $1,2, \ldots, \frac{n}{2}+$ 2 respectively and attaching $\frac{n}{2}-2$ vertices $v_{\frac{n}{2}+3}, v_{\frac{n}{2}+4}, \ldots, v_{n}$ which are labeled as $\frac{n}{2}+3, \frac{n}{2}+4, \ldots, n$ respectively, to the vertex $v_{1}$, we see that $e_{f}(0)=\frac{n}{2}$ and $e_{f}(1)=\frac{n}{2}-1$ and hence $\left|e_{f}(0)-e_{f}(1)\right| \leq 1$. Thus the resultant graph $G$ is square divisor cordial. Similarly we can construct a graph for $n$ is odd.

Theorem 2.5 can be illustrated in the following example.

Example 2. (1) $n$ is even. Let $n=12$.

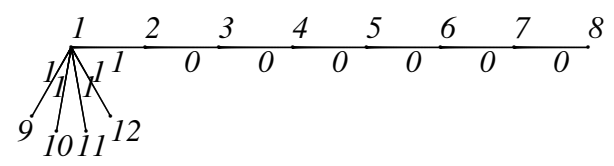

Here $e_{f}(0)=6$ and $e_{f}(1)=5$. Hence $\left|e_{f}(0)-e_{f}(1)\right|=1$.

(2) $n$ is odd. Let $n=9$.

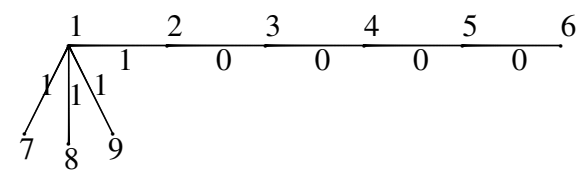

Here $e_{f}(0)=e_{f}(1)=4$ and $\left|e_{f}(0)-e_{f}(1)\right|=0$.

Theorem 2. If $G$ is a square divisor cordial graph of even size, then $G-e$ is also square divisor cordial for all e $\in E(G)$.

PROOF. Let $q$ be the even size of the square divisor cordial graph $G$. Then it follows that $e_{f}(0)=e_{f}(1)=\frac{q}{2}$. Let e be any edge in $G$ which is labeled either 0 or 1 . Then in $G-e$, we have either $e_{f}(0)=e_{f}(1)+1$ or $e_{f}(1)=e_{f}(0)+1$ and hence $\left|e_{f}(0)-e_{f}(1)\right| \leq 1$. Thus $G$-e is square divisor cordial.

Theorem 3. If $G$ is a square divisor cordial graph of odd size, then $G-e$ is also square divisor cordial for some e $\in E(G)$.

PROOF. Let $q$ be the odd size of the square divisor cordial graph $G$. Then it follows that either $e_{f}(0)=e_{f}(1)+1$ or $e_{f}(1)=e_{f}(0)+1$. If $e_{f}(0)=e_{f}(1)+1$, then remove the edge $e$ which is labeled as 0 and if $e_{f}(1)=e_{f}(0)+1$, then remove the edge e which is labeled 1 from $G$. then it follows that $e_{f}(0)=e_{f}(1)$. Thus $G-e$ is square divisor cordial for some $e \in E(G)$.

Theorem 4. The path $P_{n}$ is square divisor cordial if and only if $n \leq 12$.
PROOF. Let $P_{n}: v_{1} v_{2} \ldots v_{n}$ be the path.

Case $(i): n \leq 12$

The following table gives the square divisor cordial labeling of $P_{n}, n \leq 12$.

Vertex labels:

\begin{tabular}{|l|l|l|l|l|l|l|l|l|l|l|l|l|}
\hline$n$ & $v_{1}$ & $v_{2}$ & $v_{3}$ & $v_{4}$ & $v_{5}$ & $v_{6}$ & $v_{7}$ & $v_{8}$ & $v_{9}$ & $v_{10}$ & $v_{11}$ & $v_{12}$ \\
\hline 1 & 1 & & & & & & & & & & & \\
\hline 2 & 1 & 2 & & & & & & & & & & \\
\hline 3 & 1 & 2 & 3 & & & & & & & & & \\
\hline 4 & 1 & 2 & 4 & 3 & & & & & & & & \\
\hline 5 & 1 & 2 & 4 & 3 & 5 & & & & & & & \\
\hline 6 & 4 & 2 & 1 & 3 & 5 & 6 & & & & & & \\
\hline 7 & 4 & 2 & 1 & 3 & 5 & 6 & 7 & & & & & \\
\hline 8 & 4 & 2 & 8 & 1 & 3 & 5 & 6 & 7 & & & & \\
\hline 9 & 4 & 2 & 8 & 1 & 3 & 5 & 6 & 7 & 9 & & & \\
\hline 10 & 4 & 2 & 8 & 1 & 3 & 9 & 5 & 6 & 7 & 10 & & \\
\hline 11 & 4 & 2 & 8 & 1 & 3 & 9 & 5 & 6 & 7 & 10 & 11 & \\
\hline 12 & 4 & 2 & 8 & 1 & 3 & 9 & 5 & 6 & 7 & 10 & 11 & 12 \\
\hline
\end{tabular}

Case(ii): $n \geq 13$

If possible, let there be a square divisor cordial labeling $f$. Suppose $n$ is odd. Then obviously $e_{f}(i)=\frac{n-1}{2}, i=0,1$.

In order to get the labels 1, atmost two numbers can be assigned to the vertices that are adjacent to the vertices labeled with $1,2, \ldots,\lfloor\sqrt{n}\rfloor-1$ and atmost one number can be assigned to the vertex that is adjacent to the vertex labeled with $\lfloor\sqrt{n}\rfloor$. Therefore we have

$$
\begin{aligned}
e_{f}(1) & \leq 2(\lfloor\sqrt{n}\rfloor-1)+1 \\
\Rightarrow e_{f}(1) & \leq 2\lfloor\sqrt{n}\rfloor-1
\end{aligned}
$$

Hence

$$
\begin{aligned}
e_{f}(0) & \geq n-1-(2\lfloor\sqrt{n}\rfloor-1) \\
& =n-2\lfloor\sqrt{n}\rfloor \\
& >\frac{n-1}{2}, \quad \text { a contradiction. }
\end{aligned}
$$

Thus if $n$ is odd, there can not be a square divisor cordial labeling.

Similarly if $n$ is even, either $e_{f}(0)=\frac{n}{2}$ and $e_{f}(1)=\frac{n}{2}-1$ or $e_{f}(1)=\frac{n}{2}$ and $e_{f}(0)=\frac{n}{2}-1$ and hence there can not be $a$ square divisor cordial labeling.

Theorem 5. The cycle $C_{n}$ is square divisor cordial if and only if $3 \leq n \leq 11$.

Proof. Let $C_{n}: v_{1} v_{2} \ldots v_{n} v_{1}$ be the cycle.

Case $(i): 3 \leq n \leq 11$

The square divisor cordial labeling of $C_{n}, 3 \leq n \leq 11$ is given below.

\begin{tabular}{|l|l|l|l|l|l|l|l|l|l|l|l|}
\hline$n$ & $v_{1}$ & $v_{2}$ & $v_{3}$ & $v_{4}$ & $v_{5}$ & $v_{6}$ & $v_{7}$ & $v_{8}$ & $v_{9}$ & $v_{10}$ & $v_{11}$ \\
\hline 3 & 1 & 2 & 3 & & & & & & & & \\
\hline 4 & 1 & 2 & 3 & 4 & & & & & & & \\
\hline 5 & 1 & 2 & 4 & 3 & 5 & & & & & & \\
\hline 6 & 1 & 2 & 4 & 3 & 5 & 6 & & & & & \\
\hline 7 & 1 & 2 & 4 & 3 & 5 & 6 & 7 & & & & \\
\hline 8 & 1 & 3 & 5 & 6 & 7 & 4 & 2 & 8 & & & \\
\hline 9 & 1 & 3 & 5 & 6 & 7 & 9 & 4 & 2 & 8 & & \\
\hline 10 & 1 & 3 & 9 & 5 & 6 & 7 & 10 & 4 & 2 & 8 & \\
\hline 11 & 1 & 3 & 9 & 5 & 6 & 7 & 10 & 11 & 4 & 12 & 8 \\
\hline
\end{tabular}

Case(ii): $n \geq 12$

If there is a square divisor cordial labeling $f$, then proceeding as in the case of path, we get contradiction. 
Theorem 6. The wheel graph $W_{n}=K_{1}+C_{n-1}$ is square divisor cordial.

PROOF. Let $v_{1}$ be the central vertex and $v_{2}, v_{3}, \ldots, v_{n}$ be the vertices of $C_{n-1}$.

Define

$$
f\left(v_{1}\right)=1
$$

and

$$
f\left(v_{i}\right)=i, 2 \leq i \leq n
$$

We see that $e_{f}(0)=e_{f}(1)=n-1$. Hence $\left|e_{f}(0)-e_{f}(1)\right|=0$. Thus $W_{n}$ is square divisor cordial.

Theorem 2.11 can be illustrated in the following example.

Example 3. Let $n=11$

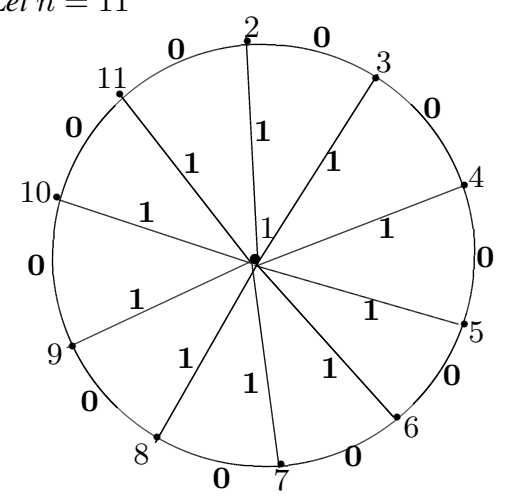

We see that $e_{f}(0)=e_{f}(1)=10$.

Theorem 7. The star graph $K_{1, n}$ is square divisor cordial if and only if $n=2,3,4,5$ or 7 .

PROOF. Let $v$ be the apex vertex and let $v_{1}, v_{2}, \ldots, v_{n}$ be the pendant vertices of the star $K_{1, n}$.

Case(i): $n=2,3,4,5$ or 7

Assign the label 2 to the vertex $v$ and the remaining labels to the vertices $v_{1}, v_{2}, \ldots, v_{n}$. If $n$ is even, then $e_{f}(0)=e_{f}(1)$ and if $n$ is odd, then either $e_{f}(0)=e_{f}(1)+1$ or $e_{f}(1)=e_{f}(0)+1$. Thus $\left|e_{f}(0)-e_{f}(1)\right| \leq 1$ and hence $K_{1, n}$ is square divisor cordial.

\section{Case(ii): $n=6$}

By labeling the vertices as in case(i), we see that $\left|e_{f}(0)-e_{f}(1)\right|=2$ and hence $K_{1, n}$ is not square divisor cordial.

\section{Case(iii): $n \geq 8$}

By labeling the vertices as in case $(i)$, we see that $\mid e_{f}(0)-$ $e_{f}(1) \mid \geq 2$ and hence $K_{1, n}$ is not square divisor cordial.

Theorem 8. The complete bipartite graph $K_{2, n}$ is square divisor cordial.

Proof. Let $V=V_{1} \cup V_{2}$ be the bipartition of $K_{2, n}$ such that $V_{1}=\left\{x_{1}, x_{2}\right\}$ and $V_{2}=\left\{y_{1}, y_{2}, \ldots, y_{n}\right\}$. Now assign the label 1 to $x_{1}$, the largest prime number $p$ such that $p \leq n+2$ to $x_{2}$ and the remaining labels to the vertices $y_{1}, y_{2}, \ldots, y_{n}$. Then it follows that $e_{f}(0)=e_{f}(1)=n$ and hence $K_{2, n}$ is square divisor cordial.

Theorem 9. The complete bipartite graph $K_{3, n}$ is square divisor cordial if and only if $n=1,2,3,5,6,7$ or 9 .
PROOF. Proof: LetV $=V_{1} \cup V_{2}$ be the bipartition of $K_{3, n}$ such that $V_{1}=\left\{x_{1}, x_{2}, x_{3}\right\}$ and $V_{2}=\left\{y_{1}, y_{2}, \ldots, y_{n}\right\}$.

Case(i): $n=1,2,3$ or 5

Assign the label 1 to $x_{1}, 2$ to $x_{2}$, the largest prime number $p$ such that $p \leq n+3$ to $x_{3}$ and the remaining labels to the vertices $y_{1}, y_{2}, \ldots, y_{n}$. If $n$ is even, then $e_{f}(0)=e_{f}(1)$ and if $n$ is odd, then either $e_{f}(0)=e_{f}(1)+1$ or $e_{f}(1)=e_{f}(0)+1$. Thus $\left|e_{f}(0)-e_{f}(1)\right| \leq 1$ and hence $K_{3, n}$ is square divisor cordial.

Case(ii): $n=6,7$ or 9

Label the vertices as in case(i) and interchange the labels $p$ and 9. If $n$ is even, then $e_{f}(0)=e_{f}(1)$ and if $n$ is odd, then $e_{f}(0)=e_{f}(1)+1$. Thus $\left|e_{f}(0)-e_{f}(1)\right| \leq 1$ and hence $K_{3, n}$ is square divisor cordial.

Case(iii): $n=4$

By labeling the vertices as in case $(i)$, we see that $\left|e_{f}(0)-e_{f}(1)\right|=2$ and hence $K_{3, n}$ is square not divisor cordial.

Case(iv): $n=8$ and $n \geq 10$

By labeling the vertices as in case(ii), we see that $\mid e_{f}(0)-$ $e_{f}(1) \mid \geq 2$ and hence $K_{3, n}$ is square not divisor cordial.

Theorem 10. The complete graph $K_{n}$ is square divisor cordial if and only if $n=1,2,3$ or 5 .

PROOF. Let $v_{1}, v_{2}, \ldots, v_{n}$ be the vertices of $K_{n}$ with $f\left(v_{i}\right)=$ $i$.

Case(i): $n=1,2,3$ or 5

The square divisor cordial labeling of $K_{1}, K_{2}, K_{3}$ and $K_{5}$ are given as follows.

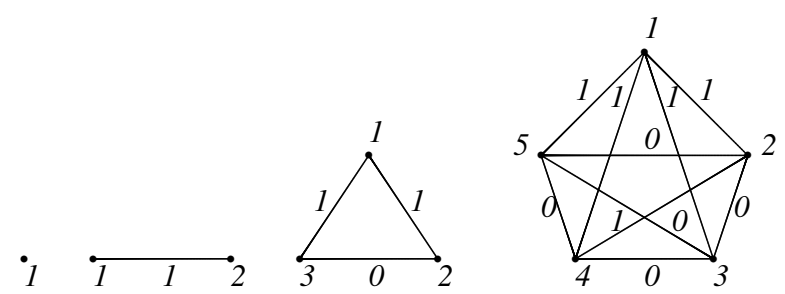

Case(ii): $n=4$

The labeling of $K_{4}$ is given as follows.

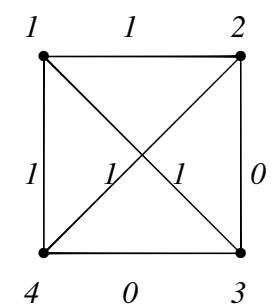

We see that $\left|e_{f}(0)-e_{f}(1)\right|=2$ and hence $K_{4}$ is not square divisor cordial.

Case(iii): $n \geq 6$

If possible, let there be a square divisor cordial labeling fof $K_{n}$. Suppose $n \equiv 0,1(\bmod 4)$.

Then obviously e $e_{f}(i)=\frac{n(n-1)}{4}, i=0,1$.

Since $v_{1}$ contributes $n-1, v_{2}$ contributes $\left\lfloor\frac{n}{2^{2}}\right\rfloor, v_{3}$ contributes 
$\left\lfloor\frac{n}{3^{2}}\right\rfloor, \ldots$ to $e_{f}(1)$, we have

$$
e_{f}(1)=n-1+\left\lfloor\frac{n}{2^{2}}\right\rfloor+\left\lfloor\frac{n}{3^{2}}\right\rfloor+\ldots+\left\lfloor\frac{n}{k^{2}}\right\rfloor,
$$

where $k$ is the largest positive integer such that $k^{2} \leq n$. Since $n \geq 6$,

$$
\begin{array}{r}
\frac{(n-1)(n-4)}{4}>\left\lfloor\frac{n}{2^{2}}\right\rfloor+\left\lfloor\frac{n}{3^{2}}\right\rfloor+\ldots+\left\lfloor\frac{n}{k^{2}}\right\rfloor \\
\Rightarrow \frac{n(n-1)}{4}-(n-1)>\left\lfloor\frac{n}{2^{2}}\right\rfloor+\left\lfloor\frac{n}{3^{2}}\right\rfloor+\ldots+\left\lfloor\frac{n}{k^{2}}\right\rfloor \\
\Rightarrow \frac{n(n-1)}{4}>n-1+\left\lfloor\frac{n}{2^{2}}\right\rfloor+\left\lfloor\frac{n}{3^{2}}\right\rfloor+\ldots+\left\lfloor\frac{n}{k^{2}}\right\rfloor \\
\Rightarrow e_{f}(1)>n-1+\left\lfloor\frac{n}{2^{2}}\right\rfloor+\left\lfloor\frac{n}{3^{2}}\right\rfloor+\ldots+\left\lfloor\frac{n}{k^{2}}\right\rfloor,
\end{array}
$$

a contradiction.

The other cases namely $n \equiv 2,3(\bmod 4)$ can be dealt with on similar lines.

\section{CONCLUDING REMARKS}

As not every divisor cordial graph is square divisor cordial it is very interesting to investigate graphs which admit square divisor cordial labeling. We have tried to investigate square divisor cor- dial labeling of standard graphs. To investigate analogous results for different graphs is an open area of research.

\section{REFERENCES}

[1] I. Cahit, Cordial graphs: A weaker version of graceful and harmonious graphs, Ars combinatoria, 23(1987), 201-207.

[2] I. Cahit, On cordial and 3-equitable labelings of graph, Utilitas Math, 370(1990), 189-198.

[3] David M. Burton, Elementary Number Theory, Second Edition,Wm. C. Brown Company Publishers, 1980.

[4] J. A. Gallian, A dynamic survey of graph labeling, Electronic Journal of Combinatorics, 17 (2010), DS6.

[5] F. Harary, Graph Theory, Addition-Wesley, Reading, Mass, 1972

[6] M. Sundaram, R. Ponraj and S, Somasundaram, Prime cordial labeling of graphs, Journal of Indian Academy of Mathematics, 27(2005), 373-390.

[7] R.Varatharajan, S.Navaneethakrishnan and K.Nagarajan, Divisor cordial graphs, International J.Math. Combin. Vol.4(2011), 15-25.

[8] R.Varatharajan, S.Navaneethakrishnan and K.Nagarajan, Special classes of divisor cordial graphs, International Mathematical Forum, Vol.7, 2012, no. 35, 1737-1749. 